\title{
AVALIAÇÃO DO BEM-ESTAR PSICOLÓGICO EM MULHERES COM HIPERTENSÃO
}

[Evaluation of psychological welfare of women with hypertension]

Nágela Valadão Cade

Eliane Corrêa Chaves *

RESUMO: Estudo descritivo, que objetivou conhecer o estado de bem-estar psicológico em mulheres hipertensas, mediante aplicação do General Health Questionnaire (GHQ) ou Questionário de Bem-Estar Psicológico de Goldberg, em 41 pacientes em tratamento para hipertensão no Instituto do Coração da FMUSP. Os resultados mostraram que as áreas relacionadas ao constructo bem-estar psicológico que mais apresentaram distúrbio foram: alteração do sono, estresse psíquico e distúrbio psicossomático. Conclui-se que as áreas com distúrbio sinalizam sofrimento psíquico e dificuldade de enfrentamento na clientela estudada, condições essas possíveis de serem trabalhadas pelo enfermeiro.

PALAVRAS-CHAVE: Bem-estar psicológico; Estado emocional; Mulheres; Hipertensão

\section{INTRODUÇÃO}

O tratamento da doença crônica objetiva reduzir os sintomas e as possíveis limitações físicas, psicológicas ou sociais que possam ocorrer e, dessa forma, fica difícil avaliar a efetividade do tratamento somente por parâmetros biológicos, até mesmo porque os tratamentos podem impor algum desconforto ao paciente, pelos efeitos colaterais das medicações, pelos custos e o tempo gasto com ele ou pela diminuição do bem-estar de forma geral.

Nessa linha de raciocínio, autores postulam quanto à necessidade de incluir, na avaliação da

*Doutora em Enfermagem, mestre em Psicologia e docente da Universidade Federal do Espírito Santo.

**Doutora em Psicologia Social, mestre em Enfermagem e docente da Escola de Enfermagem da Universidade de São Paulo. terapêutica para a doença crônica cardiovascular, critérios compreendendo a esfera social e emocional, percepção sobre a saúde e a satisfação com a vida, sendo esses indicadores considerados como "qualidade de vida" ou "bem-estar", dependendo do referencial utilizado (WENGER et al., 1984).

Ao avaliarem a qualidade de vida em portadores de doença crônica atendidos em ambulatório, constituindo os hipertensos $49,4 \%$ da amostra, concluiu-se que, para os pacientes, esse conceito associava-se à bem-estar material, bemestar físico e ao bem-estar emocional, evidenciando que os pacientes percebem esse conceito de forma mais abrangente (MARTINS et al., 1996).

As mulheres apresentam maior labilidade no bem-estar em relação aos homens, justificando a intervenção e a avaliação, nessa esfera, em clientela feminina (PASQUALI et al., 1996).

\section{OBJETIVO}

O objetivo do estudo constituiu-se em conhecer e avaliar em mulheres com hipertensão arterial, o estado de bem-estar psicológico e os seguintes fatores inter-relacionados com esse constructo: estresse psíquico, desejo de morte, falta de confiança na capacidade de desempenho, alteração do sono e distúrbio psicossomático.

\section{METODOLOGIA}

A amostra foi composta por 41 pacientes do sexo feminino portadoras de hipertensão arterial essencial, em tratamento no Instituto do Coração do Hospital das Clínicas da Faculdade de Medicina da Universidade de São Paulo.

Como critérios de inclusão da amostra, 
elegeram-se as seguintes condições: sexo feminino, ter idade acima de 21 anos, saber ler e escrever, estar em tratamento para hipertensão pelo menos há seis meses e aceitar participar do estudo, mediante assinatura do termo de consentimento pós-informado. Quanto aos critérios de exclusão da amostra, compreenderam: registro ou suspeita de hipertensão arterial secundária, outras doenças associadas à hipertensão, déficit cognitivo aparente, história de distúrbio psiquiátrico, e uso de medicação que atue diretamente no psiquismo (anfetaminas, antiepiléticos, ansiolíticos e antidepressivos).

A coleta de dados foi iniciada em 17 de fevereiro de 2000, após aprovação do projeto pelo Comitê de Ética em Pesquisa da Escola de Enfermagem da USP e pela Comissão Científica e de Ética do Instituto do Coração. As pacientes, previamente ao estudo, assinaram o Termo de Consentimento Pós- Informado.

Quanto ao procedimento, foram averiguados os prontuários das pacientes a serem consultadas na Unidade de Hipertensão, quanto à possibilidade

clínica de participarem do estudo. Quando não havia registro de características ou condições clínicas que inviabilizavam a inclusão da paciente, realizava-se uma primeira conversa com ela, na sala de espera para as consultas médicas, esclarecendo sobre a pesquisa a ser feita e confirmando os critérios de inclusão e exclusão da amostra.

Para aquelas pacientes que demonstravam interesse e disponibilidade em participar, registrava-se o nome, o número de registro da paciente na instituição e o telefone e ficava determinado um contato mais adiante.

Após seleção das pacientes, por telefone eram solicitadas à comparecerem em data, hora e local combinados para a entrevista, assinatura do termo de consentimento e preenchimento do instrumento de pesquisa.

Apesar da dificuldade em trabalhar conceitos como bem-estar por estar circunscrito a valores culturais e ter conotação ampla, sem limites muito definidos, realizou-se um recorte dessa temática com o objetivo de ser um- dos quesitos avaliados no hipertenso. O recorte referido constituiu-se na utilização de um instrumento de medida abarcando esse tipo de conteúdo.

Dessa forma, como instrumento de avaliação, utilizou-se o General Health Questionnaire (GHQ) ou Questionário de Bem-Estar Psicológico de Goldberg e um roteiro de entrevista semi-estruturada para caracterização das pacientes.

O GHQ se destina a avaliar o nível de saúde mental, a partir dos desvios em pessoas adultas da população em geral, sem história de doença psiquiátrica. Utiliza índices comportamentais observáveis, sintomas e pensamentos advindos das experiências atuais, em relação ao que geralmente ocorre, e focaliza aspectos de saúde e o bem-estar psicológico em determinado momento de vida. É respondido em escala de quatro pontos, tipo Likert (PASQUALI et al., 1996). (Anexo A)

Utilizou-se a versão de sessenta itens, constituída por cinco fatores inter-relacionados com a saúde mental: estresse psíquico, desejo de morte, desconfiança no desempenho, distúrbio do sono e distúrbio psicossomático, e um fator geral relacionado com a severidade da doença mental. Essas cinco esferas inter-relacionadas com a saúde mental, ajudam a compreendê-la e por apresentarem boa consistência interna (alfa e"0.80) no GHQ de sessenta itens, permitem serem analisadas separadamente.

Esse questionário apresenta como vantagens ser auto-aplicável e de fácil apuração por não haver necessidade de julgamento subjetivo. Foi adaptado e validado para a realidade brasileira e, apesar da validação desse instrumento ter sido aplicada em população homogênea de estudantes, considerou ser um instrumento válido e preciso para o que se propõe.Quanto maior for a pontuação obtida no GHQ, maior será o desvio da saúde mental. A pontuação alcançada em cada fator é categorizada em assintomática, sintomática ou distúrbio (PASQUALI et al., 1996).

\section{RESULTADOS}

Quanto à descrição da amostra, as pacientes apresentavam idade entre 24 a 71 anos, com média aproximada de 55 anos; a maioria era casada; não branca; ocupação do lar; renda per capita um pouco maior que dois salários mínimos; escolaridade baixa, predominando o primário completo, e faziam 
tratamento para hipertensão há aproximadamente quinze anos.

A classificação da pontuação das respostas do GHQ pode ser vista na Tabela 1.

Observa-se que, em todos os fatores, a maioria das pacientes é classificada como assintomática.

Analisando a categoria distúrbio, segundo a Tabela 1, as seguintes observações podem ser feitas:

a) o distúrbio apareceu em trinta respostas distribuídas nos seis fatores. Aqui se fala em respostas em vez de pacientes, porque uma mesma paciente pode apresentar alteração em um até seis fatores, não sendo possível estimar quantas pacientes estão envolvidas nessas respostas;

b) o fator que mais apresentou distúrbio foi o sono, acometendo onze (26,83\%) pacientes;

c) o fator que mais apresentou distúrbio, em segundo lugar, foi o estresse psíquico, observado em sete $(17,07 \%)$ pacientes; e

d) cinco $(12,19 \%)$ pacientes apresentaram distúrbio no fator distúrbio psicossomático.

As mesmas observações foram realizadas com a categoria sintomático, segundo a Tabela 1 , apresentadas a seguir:

a) os sintomas apareceram em 32 respostas distribuídas em cinco fatores; e

b) os fatores que mais apresentaram sintomas foram o fator saúde geral acometendo dez $(24,39 \%)$ pacientes e, em segundo lugar, o distúrbio psicossomático e o desejo de morte.

\section{DISCUSSÃO}

O resultado assintomático era esperado uma vez que um dos critérios de exclusão foi ser portador ou ter história de doença psiquiátrica. Nesse caso, os escores do GHQ são mais elevados, compatíveis com sintomático ou distúrbio.

Houve pacientes apresentando comprometimento nas esferas sono e estresse psíquico demonstrado por pontuações elevadas (e" 3) nesses fatores e algumas pacientes com sintomas no fator geral, o que pode representar existência de sintomatologia indicando desvio da saúde mental em relação à população feminina brasileira, segundo estudo para adaptação do GHQ (PASQUALI et al., 1996).

O GHQ foi construído baseado em escalas de depressão, ansiedade, inadequação social e hipocondria e, nesse sentido, o fator 1 , estresse psíquico, equipara-se à ansiedade, com itens relativos à tensão, irritação, impaciência, cansaço, sobrecarga e outros; o fator 2, desejo de morte, é condizente com os sintomas depressivos com ideação suicida, sentimento de desvalia e desesperança; o fator 3 , falta de confiança na capacidade de desempenho, apresenta quesitos referentes a auto-eficácia quanto à realização de tarefas diárias e (in) disposição para realizá-las; fator 4, distúrbio do sono, e o fator 5, distúrbio psicossomático, representam alterações físicas, como dor de cabeça, cansaço e outros (PASQUALI et al., 1996).

O distúrbio do sono (acordar muito cedo, perder o sono devido à preocupação, dificuldade em sua indução e manutenção e sonhos desagradáveis), quando de curta duração, associase às dificuldades, preocupações e ao estresse diário e, quando crônico, apresenta relação com quadros psiquiátricos, principalmente, a ansiedade (ROCHA, 1996).

Os dados sugeriram estar o distúrbio do sono dessa população associado ao estresse ou à tensão, pois constituíram-se as esferas com mais distúrbio e, possivelmente, esses componentes foram responsáveis pela presença de pacientes com sintomas no fator geral do GHQ, mostrando haver comprometimento nessa esfera.

... a expressão corporal constitui o primeiro, o mais primitivo meio de comunicação e de defesa de que o ser humano dispõe. É natural, portanto, que continue a utilizá-lo no decorrer da vida, sobretudo, nos

TABELA 1- FREQÜÊNCIA DA CLASSIFICAÇÃO DOS FATORES DO GHQ.

\begin{tabular}{|c|c|c|c|c|c|c|c|}
\hline \multirow{2}{*}{$\begin{array}{l}\text { Categoria } \\
\text { de resposta }\end{array}$} & Saúde Geral & Estresse Psíquico & Desejo de morte & Desconfiança desempenho & Distúrbio do sono & \multicolumn{2}{|c|}{ Distúrbio psicossomático } \\
\hline & $\%$ & $\mathrm{n} \quad \%$ & $\mathrm{n} \quad \%$ & $\%$ & $\%$ & $\mathrm{n}$ & $\%$ \\
\hline Assintomático & 2970,74 & 82,93 & 3380,48 & 82,93 & 60,98 & 29 & 70,74 \\
\hline Sintomático & $10 \quad 24,39$ & & 17,07 & 7,31 & 12,19 & 7 & 17,07 \\
\hline Distúrbio & 24,87 & 17,07 & 2,45 & 9,76 & 26,83 & 5 & 12,19 \\
\hline Total & $41 \quad 100$ & 100 & 100 & 100 & 100 & 41 & 100 \\
\hline
\end{tabular}


momentos em que outras formas de comunicação e de defesa estejam bloqueadas ou não tenham sido aprendidas (CAMPOS, 1992a, p.373).

Nesse sentido, o uso do corpo constitui uma forma menos elaborada de enfrentamento, mais primária. Considerando o nível de complexidade de enfrentamento, não foi possível acionar estratégias envolvendo abstração, cognição, elaboração e ação, que seriam mais complexas psiquicamente (CAMPOS, 1992b).

\section{CONCLUSÃO}

Este estudo mostrou, a partir do estado emocional como um todo, que as pacientes apresentaram sinais e sintomas de sofrimento psíquico e dificuldade em enfrentar as coerções cotidianas, o que entendemos como aspectos dificultadores do tratamento para hipertensão ou qualquer outro processo crônico, porque desviam o envolvimento dos pacientes com o tratamento e podem funcionar como um fator para elevação da pressão arterial.

Sugere-se, a partir dos resultados, trabalhar com esse tipo de clientela, levantando as problemáticas de vida e formas de enfrentamento mais adaptativas ao contexto das pacientes.

ABSTRACT: This is a descriptive study, and had objective of to know the psychological welfare of women with hypertension based on Goldberg Psychological Welfare applied on 41 patients treating their hypertension in the Instituto do Coração FMUSP. The results showed sleep disturbance, psychic stress and psychosomatic disturbance are Qualidade de vida de pessoas com doença crônica. Rev LatAm Enferm, Ribeirão Preto: v. 4, nº 3, 1996.

KEY WORDS: psychological welfare; emotional state; women; hypertension.

\section{REFERÊNCIAS}

1. WENGER, N. K. et al. Assessment of quality of life in clinical trials of cardiovascular therapies. Am J Cardiol., New York, v. 54, n. 1, p. 908-13. 1984. 2. PASQUALI, L. et al. QSG - Questionário de saúde geral de Goldberg: adaptação brasileira.
São Paulo: Casa do Psicólogo, 1996.

3. ROCHA, F. L. Insônia e ansiedad. In: REIMÃO, R. Sono estudo abrangente. São Paulo: Atheneu, 1996. p. 221-7.

4. ZEGANS, L. S. Stress and the developement of somatic disorders. In: OLDBERGER, L., BREZNITZ, S. Handbook of stress: theoretical and clinical aspects. New York: Free Press, 1986. p. 134-52.

5. CAMPOS, E. P. O paciente somático no grupo terapêutico. In: MELLO FILHO, J. Psicossomática hoje. Porto Alegre: Artes Médicas, 1992a. p. 37185.

6. CAMPOS E. P. Aspectos somáticos em cardiologia. In: MELLO FILHO, J. Psicossomática hoje. Porto Alegre: Artes Médicas, 1992b. p. 23458.

7. MARTINS, S. L., FRANÇA, A.P.D., KIMURA, M. Qualidade da vida de pessoas com doença crônica. Rev Lat Am Enferm. Ribeirão Preto: v. 4, n. 3, 1996.

\section{ANEXO A}

(GENERAL HEALTH QUESTIONNAIRE DE GOLDBERG)

VOCÊ ULTIMAMENTE.......

1) Tem se sentido perfeitamente bem e com boa saúde?

a) melhor do que de costume

b) como de costume

c) pior do que de costume

d) muito pior do que de costume

2) Tem sentido necessidade de tomar fortificantes (vitaminas)?
a) não, absolutamente
b) não mais do que de costume
c) um pouco mais do que de costume
d) muito mais do que de costume

3) Tem se sentido cansada (fatigada) e irritadiça?
a) não, absolutamente
b) não mais do que de costume
c) um pouco mais do que de costume
d) muito mais do que de costume

4) Tem se sentido mal de saúde? 

a) não, absolutamente
b) não mais do que de costume
c) um pouco mais do que de costume
d) muito mais do que de costume

5) Tem sentido dores de cabeça?
a) não, absolutamente
b) não mais do que de costume
c) um pouco mais do que de costume
d) muito mais do que de costume

6) Tem sentido dores de cabeça?
a) não, absolutamente
b) não mais do que de costume
c) um pouco mais do que de costume
d) muito mais do que de costume

7) Tem sido capaz de concentrar-se no que faz?
a) não, absolutamente
b) não mais do que de costume
c) um pouco mais do que de costume
d) muito mais do que de costume

8) Tem sentido medo de que você vai desmaiar num lugar público?
a) não, absolutamente
b) não mais do que de costume
c) um pouco mais do que de costume
d) muito mais do que de costume

9) Tem sentido sensações (ondas) de calor ou de frio pelo corpo?
a) não, absolutamente
b) não mais do que de costume
c) um pouco mais do que de costume
d) muito mais do que de costume

10) Tem suado (transpirado) muito?
a) não, absolutamente
b) não mais do que de costume
c) um pouco mais do que de costume
d) muito mais do que de costume

11) Tem acordado cedo (antes da hora) e não tem conseguido dormir de novo?
a) não, absolutamente
b) não mais do que de costume
c) um pouco mais do que de costume
d) muito mais do que de costume

12) Tem levantado sentindo que o sono não foi suficiente para lhe renovar as energias?
a) não, absolutamente
b) não mais do que de costume
c) um pouco mais do que de costume
d) muito mais do que de costume

13) Tem se sentido muito cansada e exausta até mesmo para alimentar-se?
a) não, absolutamente
b) não mais do que de costume
c) um pouco mais do que de costume
d) muito mais do que de costume

14) Tem perdido muito sono por causa de preocupações?
a) não, absolutamente
b) não mais do que de costume
c) um pouco mais do que de costume
d) muito mais do que de costume

15) Tem se sentido lúcida e com plena disposição mental?
a) melhor do que de costume
b) como de costume
c) menos lúcido do que de costume
d) muito menos lúcido do que de costume

16) Tem se sentido cheia de energia (com muita disposição)?
a) melhor do que de costume
b) como de costume
c) com menos energia do que de costume
d) com muito menos energia do que de costume

17) Tem sentido dificuldade em conciliar o sono (pegar no sono)?
a) não, absolutamente
b) não mais do que de costume
c) um pouco mais do que de costume
d) muito mais do que de costume

18) Tem tido dificuldade em permanecer dormindo após ter conciliado o sono (ter pego no sono)?
a) não, absolutamente
b) não mais do que de costume
c) um pouco mais do que de costume
d) muito mais do que de costume

19) Tem tido sonhos desagradáveis ou aterrorizantes?
a) não, absolutamente
b) não mais do que de costume
c) um pouco mais do que de costume
d) muito mais do que de costume

20) Tem tido noites agitadas e mal dormidas?
a) não, absolutamente
b) não mais do que de costume
c) um pouco mais do que de costume
d) muito mais do que de costume

21) Tem conseguido manter-se em atividade e ocupada? 

a) mais do que de costume
b) como de costume
c) um pouco menos do que de costume
d) muito menos do que de costume

22) Tem gasto mais tempo para executar seus afazeres?
a) mais rápido do que de costume
b) como de costume
c) mais tempo do que de costume
d) muito mais tempo do que de costume

23) Tem sentido que perde o interesse nas suas atividades normais diárias?
a) não, absolutamente
b) não mais do que de costume
c) um pouco mais do que de costume
d) muito mais do que de costume

24) Tem sentido que está perdendo interesse na sua aparência pessoal?
a) não, absolutamente
b) não mais do que de costume
c) um pouco mais do que de costume
d) muito mais do que de costume

25) Tem tido menos cuidado com suas roupas?
a) mais cuidado do que de costume
b) como de costume
c) menos cuidado do que de costume
d) muito menos cuidado do que de costume

26) Tem saído de casa com a mesma freqüência de costume?
a) mais do que de costume
b) como de costume
c) menos do que de costume
d) muito menos do que de costume

27) Tem se saído tão bem quanto acha que a maioria das pessoas sairia se estivesse em seu lugar?
a) melhor do que de costume
b) mais ou menos igual
c) um pouco pior
d) muito pior

28) Tem achado que de um modo geral tem dado boa conta de seus afazeres?
a) melhor do que de costume
b) como de costume
c) pior do que de costume
d) muito pior do que de costume

29) Tem se atrasado para chegar ao trabalho ou para começar seu trabalho em casa?

a) não, absolutamente
b) não mais do que de costume
c) um pouco mais do que de costume
d) muito mais do que de costume

30) Tem se sentido satisfeita com a forma pelo qual você tem realizado suas atividades (tarefas ou trabalho)?
a) mais satisfeito do que de costume
b) como de costume
c) menos satisfeito do que de costume
d) muito menos satisfeito do que de costume

31) Tem sido capaz de sentir calor humano e afeição por aqueles que o cercam?
a) mais do que de costume
b) como de costume
c) menos do que de costume
d) muito menos do que de costume

32) Tem achado fácil conviver com outras pessoas?
a) mais fácil do que de costume
b) tão fácil como de costume
c) mais difícil do que de costume
d) muito mais difícil do que de costume

33) Tem gasto muito tempo batendo papo?
a) mais tempo do que de costume
b) tanto quanto de costume
c) menos do que de costume
d) muito menos do que de costume

34) Tem tido medo de dizer alguma coisa as pessoas e passar por tola (parecer ridícula)?
a) não, absolutamente
b) não mais do que de costume
c) um pouco mais do que de costume
d) muito mais do que de costume

35) Tem sentido que está desempenhando uma função útil na vida?
a) mais do que de costume
b) como de costume
c) menos útil do que de costume
d) muito menos do que de costume

36) Tem se sentido capaz de tomar decisões sobre suas coisas?
a) mais do que de costume
b) como de costume
c) menos do que de costume
d) muito menos do que de costume

37) Tem sentido que você não consegue continuar as coisas que começa?
a) não, absolutamente
b) não mais do que de costume
c) um pouco mais do que de costume 
d) muito mais do que de costume

38) Tem se sentido com medo de tudo que tem que fazer?
a) não, absolutamente
b) não mais do que de costume
c) um pouco mais do que de costume
d) muito mais do que de costume

39) Tem se sentido constantemente com tensão?
a) não, absolutamente
b) não mais do que de costume
c) um pouco mais do que de costume
d) muito mais do que de costume

40) Tem se sentido incapaz de superar suas dificuldades?
a) não, absolutamente
b) não mais do que de costume
c) um pouco mais do que de costume
d) muito mais do que de costume

41) Tem achado a vida uma luta constante?
a) não, absolutamente
b) não mais do que de costume
c) um pouco mais do que de costume
d) muito mais do que de costume

42) Tem conseguido sentir prazer em suas atividades?
a) mais do que de costume
b) como de costume
c) um pouco menos do que de costume
d) muito menos do que de costume

43) Tem tido pouca paciência com as coisas?
a) não, absolutamente
b) não mais do que de costume
c) um pouco mais do que de costume
d) muito mais do que de costume

44) Tem se sentido irritada e mal humorada?
a) não, absolutamente
b) não mais do que de costume
c) um pouco mais do que de costume
d) muito mais do que de costume

45) Tem ficado apavorada ou em pânico sem razões justificadas para isso?
a) não, absolutamente
b) não mais do que de costume
c) um pouco mais do que de costume
d) muito mais do que de costume

46) Tem se sentido capaz de enfrentar problemas?
a) mais capaz do que de costume
b) como de costume
c) menos capaz do que de costume

d) muito menos capaz do que de costume 47) Tem sentido que suas atividades têm sido excessivas para você?
a) não, absolutamente
b) não mais do que de costume
c) um pouco mais do que de costume
d) muito mais do que de costume

48) Tem tido a sensação de que as pessoas olham para você?
a) não, absolutamente
b) não mais do que de costume
c) um pouco mais do que de costume
d) muito mais do que de costume

49) Tem se sentido infeliz e deprimida?
a) não, absolutamente
b) não mais do que de costume
c) um pouco mais do que de costume
d) muito mais do que de costume

50) Tem perdido a confiança em você mesma?
a) não, absolutamente
b) não mais do que de costume
c) um pouco mais do que de costume
d) muito mais do que de costume

51) Tem se considerado como uma pessoa inútil (sem valor)?
a) não, absolutamente
b) não mais do que de costume
c) um pouco mais do que de costume
d) muito mais do que de costume

52) Tem sentido que a vida é completamente sem esperança?
a) não, absolutamente
b) não mais do que de costume
c) um pouco mais do que de costume
d) muito mais do que de costume

53) Tem sentido esperançosa quanto ao seu futuro?
a) mais do que de costume
b) como de costume
c) menos do que de costume
d) muito menos do que de costume

54) Considerando-se todas as coisas, tem se sentido razoavelmente feliz?
a) mais do que de costume
b) assim como de costume
c) menos do que de costume
d) muito menos do que de costume

55) Tem se sentido nervosa e sempre tensa?
a) não, absolutamente
b) não mais do que de costume 
c) um pouco mais do que de costume

d) muito mais do que de costume

56) Tem sentido que a vida não vale a pena?

a) não, absolutamente

b) não mais do que de costume

c) um pouco mais do que de costume

d) muito mais do que de costume

57) Tem pensado na possibilidade de dar um fim em você mesma?
a) definitivamente não
b) acho que não
c) passou-me pela cabeça
d) definitivamente sim

58) Tem achado algumas vezes que não pode fazer nada porque está muito mal dos nervos?
a) não, absolutamente
b) não mais do que de costume
c) um pouco mais do que de costume
d) muito mais do que de costume

59) Já se descobriu desejando estar morta e longe (livre de tudo)?
a) não, absolutamente
b) não mais do que de costume
c) um pouco mais do que de costume
d) muito mais do que de costume

60) Tem achado que a idéia de acabar com a própria vida tem se mantido em sua mente?
a) definitivamente não
b) acho que não
c) passou-me pela cabeça
d) definitivamente sim

ENDEREÇO DASAUTORAS: Av: Carlos Orlando de Carvalho 781/ 204 Jardim da Penha 29060-260 Vitória - ES tel: 27-315 8442 e-mail: nagelavc@terra.com.br 